\title{
Epi Mönch type maps in the weak topology setting
}

\section{Donal O'Regan}

School of Mathematics, Statistics and Applied Mathematics, National University of Ireland, Galway, Ireland.

\begin{abstract}
In this paper we present coincidence, homotopy and normalization type results in the weak topology setting for general classes of Mönch type maps.
\end{abstract}

Keywords: Epi maps, coincidence, homotopy, normalization.

2010 MSC: 47H10, 54H25, 55M20.

(C)2020 All rights reserved.

\section{Introduction}

Epi maps were introduced by Furi, Martelli and Vignoli [2] and extended by many authors in the literature; see for example [3,5] and the references therein. In this paper we introduce the notion of a 0-epi (and more generally a 0-genepi) map for a general class of maps in the weak topology setting (some initial results were obtained by the author [6] in 1999). We will use 0-epi and 0-genepi maps to present coincidence, homotopy and normalization properties for general Mönch type maps (these are maps which generalize compact, condensing, countable condensing maps (in the weak topology setting) and were introduced by Mönch [4]).

\section{Main results}

Let $X$ be a Hausdorff locally convex topological vector space and $U$ a weakly open subset of $X$. We consider classes $\mathbf{A}$ and $\mathbf{B}$ of maps.

Definition 2.1. We say $F \in M\left(\overline{U^{w}}, X\right)$ (respectively $F \in M B\left(\overline{U^{w}}, X\right)$ ) if $F: \overline{U^{w}} \rightarrow 2^{X}$ and $F \in A\left(\overline{U^{w}}, X\right)$ (respectively $F \in \mathbf{B}\left(\overline{\mathrm{U}}^{w}, X\right)$ ); here $\overline{\mathrm{U}}^{w}$ denotes the weak closure of $\mathrm{U}$ in $\mathrm{X}$ and $2^{\mathrm{X}}$ denotes the family of nonempty subsets of $X$.

\section{Definition 2.2.}

(i). We say $F \in M B^{M}\left(\overline{U^{w}}, X\right)$ if $F \in M B\left(\overline{U^{w}}, X\right)$ and if $D \subseteq \overline{U^{w}}$ and $D \subseteq \overline{c o}(\{0\} \cup F(D))$ with $C \subseteq D$ countable and $C \subseteq \overline{\mathrm{co}}(\{0\} \cup \mathrm{F}(\mathrm{C}))$ then $\overline{\mathrm{C}^{w}}$ is weakly compact.

Email address: donal .oregan@nuigalway.ie (Donal O'Regan)

doi: $10.22436 /$ jnsa.013.06.02

Received: 2019-09-01 Revised: 2019-10-21 Accepted: 2020-03-03 
(ii). We say $G \in M^{M M}(\Omega, X)$ (here $\left.\Omega \subseteq X\right)$ if $G \in M B(\Omega, X)$ and if $D \subseteq \Omega, D=\overline{c o}(\{0\} \cup G(D))$ with $\mathrm{C} \subseteq \mathrm{D}$ countable and $\mathrm{C} \subseteq \overline{\mathrm{co}}(\{0\} \cup \mathrm{G}(\mathrm{C}))$ (or $\left.\overline{\mathrm{C}^{w}}=\overline{\mathrm{co}}(\{0\} \cup \mathrm{G}(\mathrm{C}))\right)$ then $\overline{\mathrm{C}^{w}}$ is weakly compact.

Remark 2.3.

(i). In Definition 2.2 (and throughout the paper) we could replace $\{0\}$ with $\left\{x_{0}\right\}$ where $x_{0} \in X$ is fixed.

(ii). In this paper we could replace the first sentence in Section 2 (Let $X$ be a Hausdorff locally convex topological vector space and $\mathrm{U}$ a weakly open subset of $\mathrm{X}$ ) with: Let $\mathrm{Y}$ be a Hausdorff locally convex topological vector space and $U$ a weakly open subset of $X$ where $X$ is a closed convex subset of $Y$ (or alternatively assume $\mathrm{X}$ is a weakly closed subset of $\mathrm{Y}$ ).

Definition 2.4. We say $F \in M_{\partial u}\left(\overline{U^{w}}, X\right)$ if $F \in M\left(\overline{U^{w}}, X\right)$ with $0 \notin F(x)$ for $x \in \partial U$; here $\partial U$ denotes the weak boundary of $U$ in $X$.

Definition 2.5. We say $F \in M B_{0}^{M}\left(\overline{U^{w}}, X\right)$ if $F \in M B^{M}\left(\overline{U^{w}}, X\right)$ and $F(x)=\{0\}$ for $x \in \partial U$.

Definition 2.6. Let $F \in M_{\partial u}\left(\overline{U^{w}}, X\right)$. We say $F$ is 0 -epi if for any map $G \in M B_{0}^{M}\left(\overline{U^{w}}, X\right)$ there exists a $x \in U$ with $F(x) \cap G(x) \neq \emptyset$.

Remark 2.7.

(i). If the zero map 0 is in $\mathrm{MB}\left(\overline{\mathrm{U}^{w}}, \mathrm{X}\right)$ then if $\mathrm{F} \in \mathrm{M}_{\partial \mathrm{U}}\left(\overline{\mathrm{U}^{w}}, \mathrm{X}\right)$ is 0 -epi then there exists a $\mathrm{x} \in \mathrm{U}$ with $0 \in \mathrm{F}(\mathrm{x})$ [Take $\mathrm{G}=0$ in Definition 2.6; note $0 \in \mathrm{MB}^{M}\left(\overline{\mathrm{U}}^{w}, X\right)$ since if $\mathrm{D} \subseteq \overline{\mathrm{U}}^{w}$ and $\mathrm{D} \subseteq \overline{\mathrm{co}}(\{0\} \cup 0(\mathrm{D}))$ with $C \subseteq D$ countable and $C \subseteq \overline{c 0}\left(\{0\} \cup 0(C)\right.$ ) (note $0(x)=0$ for $x \in C$ ) then trivially $\overline{C^{w}}$ is weakly compact, and also note trivially $\left.0 \in \mathrm{MB}_{0}^{\mathrm{M}}\left(\overline{\mathrm{U}^{w}}, \mathrm{X}\right)\right]$.

(ii). In Definition 2.4 and Definition 2.5 (and throughout the paper) we could replace 0 with $p$ where $p \in X$ is fixed.

Our first result is a coincidence property for 0-epi maps.

Theorem 2.8. Let $\mathrm{X}$ be a Hausdorff locally convex topological vector space, $\mathrm{U}$ a weakly open subset of $\mathrm{X}, 0 \in$ $\mathrm{MB}\left(\overline{\mathrm{U}^{w}}, \mathrm{X}\right), \mathrm{G} \in \mathrm{MB}^{\mathrm{M}}\left(\overline{\mathrm{U}^{w}}, \mathrm{X}\right), \mathrm{F} \in \mathrm{M}_{\partial \mathrm{U}}\left(\overline{\mathrm{U}^{w}}, \mathrm{X}\right)$ is 0-epi and suppose the following conditions hold:

$$
\left\{\begin{array}{l}
\mu(.) \mathrm{G}(.) \in \mathrm{MB}\left(\overline{\mathrm{U}^{w}}, \mathrm{X}\right) \text { for any weakly } \\
\text { continuous map } \mu: \overline{\mathrm{U}}^{w} \rightarrow[0,1] \text { with } \mu(\partial \mathrm{U})=0
\end{array}\right.
$$

and

$$
\left\{\begin{array}{l}
\mathrm{K}=\left\{x \in \overline{\mathrm{U}^{w}}: \mathrm{F}(\mathrm{x}) \cap \mathrm{t} \mathrm{G}(\mathrm{x}) \neq \emptyset \text { for some } \mathrm{t} \in[0,1]\right\} \\
\text { is weakly compact and } \mathrm{K} \text { does not intersect } \partial \mathrm{U} .
\end{array}\right.
$$

Then there exists a $\mathrm{x} \in \mathrm{U}$ with $\mathrm{F}(\mathrm{x}) \cap \mathrm{G}(\mathrm{x}) \neq \emptyset$.

Proof. Let $\mathrm{K}$ be as in the statement of Theorem 2.8. First note $\mathrm{K} \neq \emptyset$ since $\mathrm{F}$ is 0 -epi (see Remark 2.7 (i)). From (2.2) we see that $K$ is weakly compact and $K \cap \partial U=\emptyset$. Now recall $X=(X, w)$, the space $X$ endowed with the weak topology, is completely regular. Thus there exists a weakly continuous map $\mu: \overline{U^{w}} \rightarrow[0,1]$ with $\mu(\partial \mathrm{U})=0$ and $\mu(K)=1$. Define the mapping $J$ by $J(x)=\mu(x) G(x)$. Now (2.1) guarantees that $\mathrm{J} \in \mathrm{MB}\left(\overline{\mathrm{U}^{w}}, \mathrm{X}\right)$. We claim $\mathrm{J} \in \mathrm{MB}^{\mathrm{M}}\left(\overline{\mathrm{U}^{w}}, \mathrm{X}\right)$. To see this let $\mathrm{D} \subseteq \overline{\mathrm{U}^{w}}, \mathrm{D} \subseteq \overline{\mathrm{co}}(\{0\} \cup \mathrm{J}(\mathrm{D}))$ with $\mathrm{C} \subseteq \mathrm{D}$ countable and $C \subseteq \overline{c o}(\{0\} \cup \mathrm{J}(\mathrm{C}))$. Note $\mathrm{J}(\mathrm{C}) \subseteq \operatorname{co}(\{0\} \cup \mathrm{G}(\mathrm{C})), \mathrm{J}(\mathrm{D}) \subseteq \mathrm{co}(\{0\} \cup \mathrm{G}(\mathrm{D}))$ so

$$
\overline{\mathrm{co}}(\{0\} \cup \mathrm{J}(\mathrm{D})) \subseteq \overline{\mathrm{co}}(\{0\} \cup \operatorname{co}(\{0\} \cup \mathrm{G}(\mathrm{D})))=\overline{\mathrm{co}}(\operatorname{co}(\{0\} \cup \mathrm{G}(\mathrm{D})))=\overline{\mathrm{co}}(\{0\} \cup \mathrm{G}(\mathrm{D}))
$$

and $\overline{c o}(\{0\} \cup \mathrm{J}(\mathrm{C})) \subseteq \overline{\mathrm{co}}(\{0\} \cup \mathrm{G}(\mathrm{C}))$. Thus

$$
\mathrm{D} \subseteq \overline{\mathrm{co}}(\{0\} \cup \mathrm{J}(\mathrm{D})) \subseteq \overline{\mathrm{co}}(\{0\} \cup \mathrm{G}(\mathrm{D})) \text { and } \mathrm{C} \subseteq \overline{\mathrm{co}}(\{0\} \cup \mathrm{G}(\mathrm{C})) .
$$

Then since $G \in M B^{M}\left(\overline{U^{w}}, X\right)$ we have that $\overline{C^{w}}$ is weakly compact. Thus $J \in M B^{M}\left(\overline{U^{w}}, X\right)$. In fact $J \in M B_{0}^{M}\left(\overline{U^{w}}, X\right)$ since if $x \in \partial U$ we have $J(x)=\mu(x) G(x)=\{0\}$. Now since $F$ is 0 -epi there exists a $x \in U$ with $F(x) \cap J(x) \neq \emptyset$ i.e., $F(x) \cap \mu(x) G(x) \neq \emptyset$. Thus $x \in K$ and as a result $\mu(x)=1$. Consequently $\mathrm{F}(\mathrm{x}) \cap \mathrm{G}(\mathrm{x}) \neq \emptyset$. 
Our next result is a homotopy type property for 0-epi maps.

Theorem 2.9. Let $\mathrm{X}$ be a Hausdorff locally convex topological vector space, $\mathrm{U}$ a weakly open subset of $\mathrm{X}, \mathrm{F} \in$ $\mathrm{M}_{\partial \mathrm{u}}\left(\overline{\mathrm{U}^{w}}, \mathrm{X}\right)$ is $0-e p i, \mathrm{H}: \overline{\mathrm{U}^{w}} \times[0,1] \rightarrow 2^{\mathrm{X}}$ with $\mathrm{H}(\mathrm{x}, 0)=\{0\}$ for $\mathrm{x} \in \partial \mathrm{U}, \mathrm{F}()-.\mathrm{H}(., 1) \in \mathrm{M}\left(\overline{\mathrm{U}^{w}}, \mathrm{X}\right)$ and

$$
\left\{x \in \overline{\mathrm{U}^{w}}: \mathrm{F}(\mathrm{x}) \cap \mathrm{H}(\mathrm{x}, \mathrm{t}) \neq \emptyset \text { for some } \mathrm{t} \in[0,1]\right\} \text { does not intersect } \partial \mathrm{u} \text {. }
$$

Assume for any map $\mathrm{G} \in \mathrm{MB}_{0}^{\mathrm{M}}\left(\overline{\mathrm{U}^{w}}, \mathrm{X}\right)$ the following conditions hold:

$$
\begin{gathered}
\mathrm{G}(.)+\mathrm{H}(., 0) \in \mathrm{MB}^{\mathrm{M}}\left(\overline{\mathrm{U}^{w}}, \mathrm{X}\right) \\
\mathrm{K}=\left\{\mathrm{x} \in \overline{\mathrm{U}^{w}}: \mathrm{F}(\mathrm{x}) \cap[\mathrm{G}(\mathrm{x})+\mathrm{H}(\mathrm{x}, \mathrm{t})] \neq \emptyset \text { for some } \mathrm{t} \in[0,1]\right\} \text { is weakly compact }
\end{gathered}
$$

and

$$
\left\{\begin{array}{l}
\mathrm{G}(.)+\mathrm{H}(., \mu(.)) \in \mathrm{MB}^{\mathrm{M}}\left(\overline{\mathrm{U}^{w}}, \mathrm{X}\right) \text { for any weakly continuous } \\
\text { map } \mu: \overline{\mathrm{U}^{w}} \rightarrow[0,1] \text { with } \mu(\partial \mathrm{U})=0 \text { and } \mu(\mathrm{K})=1 .
\end{array}\right.
$$

Then $\mathrm{F}()-.\mathrm{H}(., 1)$ is $0-e p i$.

Proof. Let $\mathrm{G} \in \mathrm{MB}_{0}^{\mathrm{M}}\left(\overline{\mathrm{U}^{w}}, \mathrm{X}\right)$. We must show that there exists a $x \in \mathrm{U}$ with $[\mathrm{F}(\mathrm{x})-\mathrm{H}(\mathrm{x}, 1)] \cap \mathrm{G}(\mathrm{x}) \neq \emptyset$. Let $H$ and $K$ be as in the statement of Theorem 2.9. Consider $t=0$. Note from (2.4) that $G()+.H(., 0) \in$ $M_{0}^{M}\left(\overline{U^{w}}, X\right)$ since if $x \in \partial U$ then $G(x)+H(x, 0)=G(x)=\{0\}$ since $G \in M B_{0}^{M}\left(\overline{U^{w}}, X\right)$. Now since $F$ is $0-$ epi there exists a $x \in U$ with $F(x) \cap[G(x)+H(x, 0)] \neq \emptyset$, so $K \neq \emptyset$, and in addition from (2.5) we have that $K$ is weakly compact. Next note $K \cap \partial U=\emptyset$ since if $x \in \partial U$ then $F(x) \cap[G(x)+H(x, t)]=F(x) \cap H(x, t)$ and note (2.3). Now $X=(X, w)$ is completely regular so there exists a weakly continuous map $\mu: \overline{U^{w}} \rightarrow[0,1]$ with $\mu(\partial \mathrm{U})=0$ and $\mu(K)=1$. Define the map $J$ by $J(x)=G(x)+H(x, \mu(x))$. From (2.6) we have $J \in$ $M_{0}^{M}\left(\overline{U^{w}}, X\right)$ since if $x \in \partial U$ then $J(x)=G(x)+H(x, \mu(x))=\{0\}+H(x, 0)=\{0\}$ since $G \in M B_{0}^{M}\left(\overline{U^{w}}, X\right)$. Then since $F$ is 0 -epi there exists a $x \in U$ with $F(x) \cap J(x) \neq \emptyset$ i.e., $F(x) \cap[G(x)+H(x, \mu(x))] \neq \emptyset$. Thus $x \in K$ and as a result $\mu(x)=1$. Consequently $F(x) \cap[G(x)+H(x, 1)] \neq \emptyset$ i.e., $[F(x)-H(x, 1)] \cap G(x) \neq$ $\emptyset$.

Next we present a normalization type result.

Theorem 2.10. Let $\mathrm{X}$ be a Hausdorff locally convex topological vector space, $\mathrm{U}$ a weakly open subset of $\mathrm{X}, 0 \in \mathrm{U}$ and assume the following conditions hold:

$i \in M\left(\overline{\mathrm{U}^{w}}, \mathrm{X}\right)$ (here $\mathrm{i}$ is the identity map)

$$
\left\{\begin{array}{l}
\text { for any map } \mathrm{H} \in \mathrm{MB}_{0}^{\mathrm{M}}\left(\overline{\mathrm{U}^{w}}, \mathrm{X}\right) \text { and } \\
\mathrm{J}(\mathrm{x})=\left\{\begin{array}{l}
\mathrm{H}(\mathrm{x}), \quad \mathrm{x} \in \mathrm{U} \\
\{0\}, \quad x \in X \backslash \mathrm{U},
\end{array}\right. \\
\text { we have that } \mathrm{J} \in \mathrm{MB}(\mathrm{X}, \mathrm{X})
\end{array}\right.
$$

$\left\{\begin{array}{l}\text { for any map } \mathrm{H} \in \mathrm{MB}_{0}^{\mathrm{M}}\left(\overline{\mathrm{U}^{w}}, \mathrm{X}\right) \text { and for any countable } \\ \text { set } \mathrm{P} \subseteq \mathrm{X} \text { with } \mathrm{P} \cap \overline{\mathrm{U}^{w}} \text { relatively weakly compact we } \\ \text { have that the set } \overline{\mathrm{co}}\left(\{0\} \cup \mathrm{H}\left(\mathrm{P} \cap \overline{\mathrm{U}^{w}}\right)\right) \text { is weakly compact }\end{array}\right.$

and

$$
\left\{\begin{array}{l}
\text { for any map } \mathrm{G} \in \mathrm{MB}^{\mathrm{MM}}(\mathrm{X}, \mathrm{X}) \text { there exists an } \\
\mathrm{x} \in \mathrm{X} \text { with } \mathrm{x} \in \mathrm{G}(\mathrm{x})
\end{array}\right.
$$

Then $i$ is $0-e p i$.

Proof. Note $i \in M_{\partial u}\left(\overline{U^{w}}, X\right)$ (from (2.7) and $0 \in U$ ). Let $H \in M B_{0}^{M}\left(\overline{U^{w}}, X\right)$. We must show there exits a $x \in U$ with $x \in H(x)$ (i.e., $i(x) \cap H(x) \neq \emptyset$ ). Let $J$ be as in (2.8). Note $J \in M B(X, X)$. We claim 
$\mathrm{J} \in \mathrm{MB}^{\mathrm{MM}}(\mathrm{X}, \mathrm{X})$. To see this let $\mathrm{D} \subseteq \mathrm{X}, \mathrm{D}=\overline{\mathrm{co}}(\{0\} \cup \mathrm{J}(\mathrm{D}))$ with $\mathrm{C} \subseteq \mathrm{D}$ countable and $\mathrm{C} \subseteq \overline{\mathrm{co}}(\{0\} \cup \mathrm{J}(\mathrm{C}))$ (or $\left.\overline{C^{w}}=\overline{c o}(\{0\} \cup J(C))\right)$. First note $\overline{c o}(\{0\} \cup J(D)) \subseteq \overline{c o}\left(\{0\} \cup \mathrm{H}\left(D \cap \overline{U^{w}}\right)\right)$ so $D=\overline{c o}(\{0\} \cup J(D)) \subseteq$ $\overline{\mathrm{co}}\left(\{0\} \cup \mathrm{H}\left(\mathrm{D} \cap \overline{\mathrm{U}^{w}}\right)\right)$ and $\mathrm{C} \subseteq \overline{\mathrm{co}}\left(\{0\} \cup \mathrm{H}\left(\mathrm{C} \cap \overline{\mathrm{U}^{w}}\right)\right)$. As a result

$$
\mathrm{D} \cap \overline{\mathrm{U}^{w}} \subseteq \overline{\mathrm{co}}\left(\{0\} \cup \mathrm{H}\left(\mathrm{D} \cap \overline{\mathrm{U}^{w}}\right)\right) \text { and } \mathrm{C} \cap \overline{\mathrm{U}^{w}} \subseteq \overline{\mathrm{co}}\left(\{0\} \cup \mathrm{H}\left(\mathrm{C} \cap \overline{\mathrm{U}^{w}}\right)\right) ;
$$

note $\mathrm{C} \cap \overline{\mathrm{U}^{w}}$ is countable. Now since $\mathrm{H} \in \mathrm{MB}^{\mathrm{M}}\left(\overline{\mathrm{U}}^{w}, \mathrm{X}\right)$ we have (see (2.11)) that ${\overline{\mathrm{C} \cap \overline{\mathrm{U}}^{w}}}^{w}$ is weakly compact. Now since $C \subseteq \overline{\mathrm{CO}}\left(\{0\} \cup \mathrm{H}\left(\mathrm{C} \cap \overline{\mathrm{U}^{w}}\right)\right)$ we have that $\overline{\mathrm{C}^{w}}$ is weakly compact (see (2.9)). Thus $J \in M^{M M}(X, X)$. Now (2.10) guarantees that there exists a $x \in X$ with $x \in J(x)$. In fact $x \in U$ since $0 \in U$. Thus $x \in U$ and $x \in H(x)$.

\section{Remark 2.11.}

(i). Conditions to guarantee (2.10) can be found in $[7,8]$.

(ii). In the proof above we showed $J$ in (2.8) is in $\mathrm{MB}^{M M}(X, X)$ so one could replace (2.10) with: there exists a $x \in X$ with $x \in J(x)$.

To conclude this paper we will consider a generalization of the notion of a 0-epi maps. To present our results we consider an extra class $\mathbf{D}$ of maps. Let $M$ and $M B$ be as in Definition 2.1.

Definition 2.12. We say $\mathrm{F} \in \mathrm{MD}\left(\overline{\mathrm{U}^{w}}, \mathrm{X}\right)$ if $\mathrm{F}: \overline{\mathrm{U}^{w}} \rightarrow 2^{\mathrm{X}}$ and $\mathrm{F} \in \mathrm{D}\left(\overline{\mathrm{U}^{w}}, \mathrm{X}\right)$

Definition 2.13. We say $F \in \operatorname{MBB}\left(\overline{\mathrm{U}^{w}}, X\right)$ if $F \in M B\left(\overline{U^{w}}, X\right)$ and there exists a selection $\Psi \in M D\left(\overline{U^{w}}, X\right)$ of $F$.

Remark 2.14.

(i). Note in Definition 2.13, $\Psi$ is a selection of $F$ if $\Psi(x) \subseteq F(x)$ for $x \in \overline{U^{w}}$.

(ii). In many applications the spaces $M D$ and $M B B$ are the same (i.e., $M D=M B B=M B$ ). For examples of when the spaces MD and MBB are different we refer the reader to [1] (e.g. MBB is the space of WDKT maps (see [1]) and MD is the space of weakly continuous single valued maps).

\section{Definition 2.15.}

(i). We say $\Psi \in \mathrm{MD}^{\mathrm{M}}\left(\overline{\mathrm{U}^{w}}, \mathrm{X}\right)$ if $\Psi \in \mathrm{MD}\left(\overline{\mathrm{U}^{w}}, \mathrm{X}\right)$ and if $\mathrm{D} \subseteq \overline{\mathrm{U}^{w}}$ and $\mathrm{D} \subseteq \overline{\mathrm{co}}(\{0\} \cup \Psi(\mathrm{D}))$ with $\mathrm{C} \subseteq \mathrm{D}$ countable and $C \subseteq \overline{\mathrm{co}}(\{0\} \cup \Psi(\mathrm{C}))$ then $\overline{\mathrm{C}^{w}}$ is weakly compact.

(ii). We say $F \in M_{B B}^{M}\left(\overline{U^{w}}, X\right)$ if $F \in M B\left(\overline{U^{w}}, X\right)$ and there exists a selection $\Psi \in M D^{M}\left(\overline{U^{w}}, X\right)$ of $F$.

(iii). We say $\Psi \in \operatorname{MD}^{M M}(\Omega, X)$ (here $\left.\Omega \subseteq X\right)$ if $\Psi \in M D(\Omega, X)$ and if $D \subseteq \Omega, D=\overline{c o}(\{0\} \cup \Psi(D)$ ) with $\mathrm{C} \subseteq \mathrm{D}$ countable and $\mathrm{C} \subseteq \overline{\mathrm{co}}(\{0\} \cup \Psi(\mathrm{C}))$ (or $\left.\overline{\mathrm{C}^{w}}=\overline{\mathrm{co}}(\{0\} \cup \Psi(\mathrm{C}))\right)$ then $\overline{\mathrm{C}^{w}}$ is weakly compact.

(iv). We say $F \in \operatorname{MBB}^{M M}(\Omega, X)$ (here $\left.\Omega \subseteq X\right)$ if $F \in M B(\Omega, X)$ and there exists a selection $\Psi \in$ $\operatorname{MD}^{M M}(\Omega, X)$ of $F$.

Definition 2.16. We say $F \in M_{B B}^{M}\left(\overline{U^{w}}, X\right)$ if $F \in M_{B B}^{M}\left(\overline{U^{w}}, X\right)$ and $F(x)=\{0\}$ for $x \in \partial U$.

Definition 2.17. Let $F \in M_{\partial u}\left(\overline{U^{w}}, X\right)$. We say $F$ is 0-genepi if for any map $G \in M_{B B}^{M}\left(\overline{U^{w}}, X\right)$ and any selection $\Psi \in M^{M}\left(\overline{U^{w}}, X\right)$ of $G$ there exists a $x \in U$ with $F(x) \cap \Psi(x) \neq \emptyset$.

Remark 2.18. In Definition 2.17 note $\emptyset \neq F(x) \cap \Psi(x) \subseteq F(x) \cap G(x)$. If the zero map is in $M D\left(\overline{U^{w}}, X\right)$ then if $F \in M_{\partial u}\left(\bar{U}^{w}, X\right)$ is 0 -genepi then there exists a $x \in U$ with $0 \in F(x)$; take $G=0$ in Definition 2.17 (note $0 \in \operatorname{MD}^{M}\left(\overline{\mathrm{U}^{w}}, X\right)$ using the argument in Remark $2.7(\mathrm{i})$, and also note if $\Psi \in \mathrm{MD}^{M}\left(\overline{\mathrm{U}^{w}}, \mathrm{X}\right)$ is a selection of 0 then $\Psi=0$ since $\Psi(x) \subseteq 0(x)$ for $x \in \overline{\mathrm{U}^{w}}$ and $0 \in \mathrm{MD}^{M}\left(\overline{\mathrm{U}^{w}}, \mathrm{X}\right)$ ).

We begin with an analogue of Theorem 2.8.

Theorem 2.19. Let $\mathrm{X}$ be a Hausdorff locally convex topological vector space, $\mathrm{U}$ a weakly open subset of $\mathrm{X}, 0 \in$ $\mathrm{MD}\left(\overline{\mathrm{U}^{w}}, \mathrm{X}\right), \mathrm{G} \in \mathrm{MBB}^{\mathrm{M}}\left(\overline{\mathrm{U}^{w}}, \mathrm{X}\right), \overline{\mathrm{F}} \in \mathrm{M}_{\mathrm{\partial u}}\left(\overline{\mathrm{U}^{w}}, \mathrm{X}\right)$ is 0-genepi and suppose

$$
\left\{\begin{array}{l}
\mu(.) \mathrm{G}(.) \in \operatorname{MBB}\left(\overline{\mathrm{U}^{w}}, \mathrm{X}\right) \text { for any weakly } \\
\text { continuous map } \mu: \overline{\mathrm{U}^{w}} \rightarrow[0,1] \text { with } \mu(\partial \mathrm{U})=0 .
\end{array}\right.
$$


For any selection $\Lambda \in \mathrm{MD}^{\mathrm{M}}\left(\overline{\mathrm{U}^{w}}, \mathrm{X}\right)$ of $\mathrm{G}$ assume the following hold:

$$
\left\{\begin{array}{l}
\mathrm{K}=\left\{\mathrm{x} \in \overline{\mathrm{U}^{w}}: \mathrm{F}(\mathrm{x}) \cap \mathrm{t} \Lambda(\mathrm{x}) \neq \emptyset \text { for some } \mathrm{t} \in[0,1]\right\} \\
\text { is weakly compact and } \mathrm{K} \text { does not intersect du }
\end{array}\right.
$$

and

$$
\left\{\begin{array}{l}
\mu(.) \wedge(.) \in M D\left(\overline{\mathrm{U}^{w}}, \mathrm{X}\right) \text { for any weakly continuous } \\
\text { map } \mu: \overline{\mathrm{U}^{w}} \rightarrow[0,1] \text { with } \mu(\partial \mathrm{U})=0 \text { and } \mu(\mathrm{K})=1 .
\end{array}\right.
$$

Then there exists a $x \in \mathrm{U}$ with $\mathrm{F}(\mathrm{x}) \cap \Lambda(\mathrm{x}) \neq \emptyset($ so $\emptyset \neq \mathrm{F}(\mathrm{x}) \cap \Lambda(\mathrm{x}) \subseteq \mathrm{F}(\mathrm{x}) \cap \mathrm{G}(\mathrm{x}))$.

Proof. Let $\Lambda$ and $K$ be as in the statement of Theorem 2.19. Note $K \neq \emptyset$ since $0 \in F(x)$ for some $x \in U$ (see Remark 2.18; note $\left.0 \in M D\left(\overline{\mathrm{U}^{w}}, X\right)\right)$. Now (see (2.13)) there exists a weakly continuous map $\mu: \overline{\mathrm{U}^{w}} \rightarrow[0,1]$ with $\mu(\partial \mathrm{U})=0$ and $\mu(K)=1$. Define the mappings $J$ and $\Psi$ by $J(x)=\mu(x) G(x)$ and $\Psi(x)=\mu(x) \Lambda(x)$. Now (2.14) guarantees that $\Psi \in M D\left(\overline{\mathrm{U}^{w}}, X\right)$. We claim $\Psi \in \operatorname{MD}^{M}\left(\overline{\mathrm{U}^{w}}, X\right)$. To see this let $\mathrm{D} \subseteq \overline{\mathrm{U}^{w}}$, $\mathrm{D} \subseteq \overline{\mathrm{co}}(\{0\} \cup \Psi(\mathrm{D}))$ with $\mathrm{C} \subseteq \mathrm{D}$ countable and $\mathrm{C} \subseteq \overline{\mathrm{co}}(\{0\} \cup \Psi(\mathrm{C}))$. Note $\Psi(\mathrm{C}) \subseteq \operatorname{co}(\{0\} \cup \Lambda(\mathrm{C}))$, $\Psi(\mathrm{D}) \subseteq \operatorname{co}(\{0\} \cup \Lambda(\mathrm{D}))$ so (a similar argument to that in Theorem 2.8)

$$
\mathrm{D} \subseteq \overline{\mathrm{co}}(\{0\} \cup \Lambda(\mathrm{D})) \text { and } \mathrm{C} \subseteq \overline{\mathrm{co}}(\{0\} \cup \Lambda(\mathrm{C})) .
$$

Now since $\Lambda \in M^{M}\left(\overline{\mathrm{U}^{w}}, X\right)$ we have that $\overline{\mathrm{C}^{w}}$ is weakly compact. Thus $\Psi \in \mathrm{MD}^{M}\left(\overline{\mathrm{U}^{w}}, \mathrm{X}\right)$. Also (2.12) guarantees that $J \in \operatorname{MBB}\left(\overline{\mathrm{U}^{w}}, X\right)$ so immediately we have $J \in \operatorname{MBB}^{M}\left(\overline{\mathrm{U}^{w}}, X\right)$ since $\Psi \in \mathrm{MD}^{M}\left(\overline{\mathrm{U}^{w}}, X\right)$ and $\Psi$ is a selection of $J$ (since $\Lambda$ is a selection of $G$ ). Finally note $J \in \operatorname{MBB}_{0}^{M}\left(\overline{U^{w}}, X\right)$ since if $x \in \partial U$ we have $J(x)=\mu(x) G(x)=\{0\}$ since $\mu(\partial U)=0$. Now since $F$ is 0 -genepi then there exists a $x \in U$ with $F(x) \cap \Psi(x) \neq \emptyset$ i.e., $F(x) \cap \mu(x) \wedge(x) \neq \emptyset$. Thus $x \in K$ and as a result $\mu(x)=1$. Consequently $\mathrm{F}(\mathrm{x}) \cap \Lambda(\mathrm{x}) \neq \emptyset$.

Next we obtain an analogue of Theorem 2.9.

Theorem 2.20. Let $\mathrm{X}$ be a Hausdorff locally convex topological vector space, $\mathrm{U}$ a weakly open subset of $\mathrm{X}, \mathrm{F} \in$ $\mathrm{M}_{\mathrm{\partial u}}\left(\overline{\mathrm{U}^{w}}, \mathrm{X}\right)$ is 0-genepi, $\mathrm{H}: \overline{\mathrm{U}^{w}} \times[0,1] \rightarrow 2^{\mathrm{X}}$ with $\mathrm{H}(\mathrm{x}, 0)=\{0\}$ for $\mathrm{x} \in \partial \mathrm{U}, \mathrm{F}()-.\mathrm{H}(., 1) \in \mathrm{M}\left(\overline{\mathrm{U}^{w}}, \mathrm{X}\right)$ and

$$
\left\{x \in \overline{\mathrm{U}^{w}}: \mathrm{F}(\mathrm{x}) \cap \mathrm{H}(\mathrm{x}, \mathrm{t}) \neq \emptyset \text { for some } \mathrm{t} \in[0,1]\right\} \text { does not intersect } \partial \mathrm{U} .
$$

Assume there exists a selection $\mathrm{h}$ of $\mathrm{H}$ such that for any $\mathrm{G} \in \mathrm{MBB}_{0}^{\mathrm{M}}\left(\overline{\mathrm{U}^{w}}, \mathrm{X}\right)$ and any selection $\Psi \in \mathrm{MD}^{\mathrm{M}}\left(\overline{\mathrm{U}^{w}}, \mathrm{X}\right)$ of $\mathrm{G}$ the following hold:

$$
\begin{gathered}
\mathrm{G}(.)+\mathrm{H}(., 0) \in \mathrm{MBB}^{\mathrm{M}}\left(\overline{\mathrm{U}^{w}}, \mathrm{X}\right) \text { and } \Psi(.)+\mathrm{h}(., 0) \in \mathrm{MD}^{\mathrm{M}}\left(\overline{\mathrm{U}^{w}}, \mathrm{X}\right) \\
\mathrm{K}=\left\{\mathrm{x} \in \overline{\mathrm{U}^{w}}: \mathrm{F}(\mathrm{x}) \cap[\Psi(\mathrm{x})+\mathrm{h}(\mathrm{x}, \mathrm{t})] \neq \emptyset \text { for some } \mathrm{t} \in[0,1]\right\} \text { is weakly compact }
\end{gathered}
$$

and

$$
\left\{\begin{array}{l}
\mathrm{G}(.)+\mathrm{H}(., \mu(.)) \in \mathrm{MBB}^{\mathrm{M}}\left(\overline{\mathrm{U}^{w}}, \mathrm{X}\right) \text { and } \Psi(.)+\mathrm{h}(., \mu(.)) \in \mathrm{MD}^{\mathrm{M}}\left(\overline{\mathrm{U}^{w}}, \mathrm{X}\right) \\
\text { for any weakly continuous map } \mu: \overline{\mathrm{U}^{w}} \rightarrow[0,1] \text { with } \mu(\mathrm{\partial U})=0 \text { and } \mu(\mathrm{K})=1 .
\end{array}\right.
$$

Then $\mathrm{F}()-.\mathrm{H}(., 1)$ is $0-$ genepi.

Proof. Let $\mathrm{G} \in \mathrm{MBB}_{0}^{M}\left(\overline{\mathrm{U}^{w}}, \mathrm{X}\right)$ and let $\Psi \in \mathrm{MD}^{\mathrm{M}}\left(\overline{\mathrm{U}^{w}}, \mathrm{X}\right)$ be any selection of $\mathrm{G}$. Now let $\mathrm{h}, \mathrm{H}$ and $\mathrm{K}$ be as in the statement of Theorem 2.20. Consider $t=0$. Note $\mathrm{G}()+.\mathrm{H}(., 0) \in \mathrm{MBB}_{0}^{M}\left(\overline{\mathrm{U}^{w}}, \mathrm{X}\right)$ and $\Psi()+.h(., 0) \in M^{M}\left(\overline{U^{w}}, X\right)$ is a selection of $G()+.H(., 0)$. Now since $F$ is 0 -genepi then there exists a $x \in U$ with $F(x) \cap[\Psi(x)+h(x, 0)] \neq \emptyset$, so $K \neq \emptyset$. Now $K \cap \partial U=\emptyset$ since if $x \in \partial U$ then $F(x) \cap[\Psi(x)+h(x, t)] \subseteq F(x) \cap[G(x)+H(x, t)]=F(x) \cap H(x, t)$ and note (2.15). Now $X=(X, w)$ is completely regular so there exists a weakly continuous map $\mu: \overline{U^{w}} \rightarrow[0,1]$ with $\mu(\partial \mathrm{U})=0$ and $\mu(K)=1$. Define maps $J$ and $\Lambda$ by $J(x)=G(x)+H(x, \mu(x))$ and $\Lambda(x)=\Psi(x)+h(x, \mu(x))$. Note we have $J \in M_{B} M\left(\overline{U^{w}}, X\right)$ (if $x \in \partial U$ then $\left.J(x)=G(x)+H(x, 0)=\{0\}\right)$ and $\Lambda \in M D^{M}\left(\overline{U^{w}}, X\right)$ is a selection of $J$. Then since $F$ is 0 -genepi there exists a $x \in U$ with $F(x) \cap \Lambda(x) \neq \emptyset$ i.e., $F(x) \cap[\Psi(x)+h(x, \mu(x))] \neq \emptyset$. Thus $x \in K$ and as a result $\mu(x)=1$. Consequently $F(x) \cap[\Psi(x)+h(x, 1)] \neq \emptyset$, so $F(x) \cap[\Psi(x)+H(x, 1)] \neq \emptyset$ i.e., $[F(x)-H(x, 1)] \cap \Psi(x) \neq \emptyset$. 
Remark 2.21. Note in Theorem 2.20 we could replace (2.15) with (here $h$ and $\Psi$ are as described in the statement of Theorem 2.20)

$$
\left\{x \in \overline{U^{w}}: F(x) \cap h(x, t) \neq \emptyset \text { for some } t \in[0,1]\right\} \text { does not intersect } \partial \mathrm{U} .
$$

It is easy to obtain analogues of Theorem 2.10 in this situation (we leave the details to the reader).

\section{References}

[1] R. P. Agarwal, D. O'Regan, Continuation methods for closed, weakly closed, DKT and WDKT maps, Comput. Math. Appl., 38 (1999), 81-88. 2.14

[2] M. Furi, M. Martelli, A. Vignoli, On the solvability of nonlinear operator equations in normed spaces, Ann. Math. Pura Appl. (4), 124 (1980), 321-343. 1

[3] G. Gabor, L. Górniewicz, M. Ślosarski, Generalized topological essentiality and coincidence points of multivalued maps, Set-Valued Var. Anal., 17 (2009), 1-19. 1

[4] H. Mönch, Boundary value problems for nonlinear ordinary differential equations in Banach spaces, Nonlinear Anal., 4 (1980), 985-999. 1

[5] D. O'Regan, Continuation methods based on essential and 0-epi maps, Acta Appl. Math., 54 (1998), 319-330. 1

[6] D. O'Regan, Weakly contractive zero epi maps, Math. Comput. Modelling, 30 (1999), 21-25. 1

[7] D. O'Regan, Maps with weakly sequentially closed graphs satisfying compactness conditions on countable sets, Pure and Applied Functional Analysis, to appear. 2.11

[8] D. O'Regan, Coincidence results for compositions of multivalued maps based on countable compactness principles, J. Nonlinear Convex Anal., to appear in 21 (2020), 7 pages. 2.11 